Cahiers $d u$ MONDE RUSSE

\section{Cahiers du monde russe}

Russie - Empire russe - Union soviétique et États indépendants

$46 / 4 \mid 2005$

L'invention d'une politique humanitaire

\title{
Yuri Slezkine, The Jewish Century
}

\section{Maurice Kriegel}

\section{OpenEdition \\ Journals}

Édition électronique

URL : https://journals.openedition.org/monderusse/6667

DOI : $10.4000 /$ monderusse. 6667

ISSN : $1777-5388$

Éditeur

Éditions de l'EHESS

Édition imprimée

Date de publication : 1 décembre 2005

Pagination : 977-979

ISBN : 2-7132-2057-2

ISSN : $1252-6576$

\section{Référence électronique}

Maurice Kriegel, «Yuri Slezkine, The Jewish Century », Cahiers du monde russe [En ligne], 46/4 | 2005, mis en ligne le 30 juin 2009, consulté le 04 septembre 2022. URL : http://journals.openedition.org/ monderusse/6667; DOI : https://doi.org/10.4000/monderusse.6667

Ce document a été généré automatiquement le 4 septembre 2022

Tous droits réservés 


\title{
Yuri Slezkine, The Jewish Century
}

\author{
Maurice Kriegel
}

\section{RÉFÉRENCE}

Yuri SLEZKINE, The Jewish Century. Princeton, Oxford : Princeton University Press, 2004, $438 \mathrm{p}$.

1 Yuri Slezkine consacre cet essai à l'histoire des Juifs russes durant l'époque soviétique, mais l'intitule de façon très générale: The Jewish Century. Il ne s'agit pas d'un coup de pouce par lequel l'auteur donnerait un titre alléchant à un ouvrage spécialisé: sa relecture de ce pan particulier de l'histoire juive contemporaine prend appui sur une interprétation globale du rôle des Juifs dans l'histoire du $\mathrm{xx}^{\mathrm{e}}$ siècle et plus largement encore des rapports entre monde juif et modernité. Le sens, le rythme de l'aventure historique des Juifs d'URSS sont quasiment déduits de la série de propositions avancées dans les premiers chapitres de l'ouvrage. Cette cohérence de la démarche fait à la fois la force et la fragilité de l'entreprise.

2 Pour saisir le fait juif dans son ensemble et, apparemment, dans le souci de lui dénier toute forme d'« unicité", Slezkine reprend la théorie classique sur les diasporas marchandes, mais marque deux inflexions. D'une part, deux traits principaux caractérisent selon lui ces diasporas installées au milieu des sociétés traditionnelles: ayant plus affaire aux hommes qu'aux choses, elles valorisent la mobilité de l'intelligence, l'adresse, l'interprétation des signes - les attributs du dieu Hermès (ou Mercure) et de ses équivalents dans les différents panthéons; indifférentes à l'espace, elles se définissent dans et par le temps, et leur obsession généalogique les transforme en groupes fermés. D'autre part, la modernité des $\mathrm{XIX}^{\mathrm{e}}-\mathrm{XX}^{\mathrm{e}}$ siècles se définit précisément par l'universalisation de ces deux traits, sous forme à peine transposée: sur le plan économique, elle crée un monde de l'échange et de la promotion des services; sur le plan politique, domine désormais le nationalisme. Les sociétés modernes se sont ainsi « mercurisées », et, du même coup, judaïsées (adversaires et défenseurs des Juifs, lorsque cristallise l'antisémitisme moderne à la fin du XIX ${ }^{e}$ siècle, sont d'accord pour estimer 
qu'ils symbolisent le moderne). La grandeur et la misère de l'histoire juive aux XIX $\mathrm{e}_{\text {- }}$ $\mathrm{xx}^{\mathrm{e}}$ siècles procèdent de la prédominance de ces deux traits : mercuriens de toujours, ils excellent dans les formes d'activité que les peuples « apolliniens » (attachés à des valeurs et des comportements typiques de vieilles civilisations agraires, et défendues par Apollon, le dieu protecteur des troupeaux), en cours de "mercurisation ", maîtrisent encore mal. D'où une explosion d'énergie et de talent, dans l'initiative économique comme dans l'ordre culturel. Mais, bénéficiaires et héros de l'âge moderne, les Juifs sont aussi ses premières victimes, puisqu'il est l'âge du nationalisme, dont le nazisme représente l'expression paroxystique. Par leurs réussites et dans la tragédie, les Juifs occupent ainsi une position centrale dans un $\mathrm{xx}^{\mathrm{e}}$ siècle qu'on peut désigner comme «le siècle juif ».

3 Pour Slezkine, à partir du dernier tiers du XIX ${ }^{\mathrm{e}}$ siècle, les Juifs de l'empire russe revivent dans le contexte particulier du régime tsariste une histoire, avec ses affres et ses chances, découpée dans la même étoffe que celle des Juifs d'Europe occidentale et centrale dans les décennies précédentes. La modernisation de la Russie périme les fonctions remplies en milieu rural par la " caste » juive et entraîne sa paupérisation; mais elle offre en même temps, pour une minorité, de formidables economic opportunities. Elle fait aussi entrer en crise le monde juif traditionnel et détermine le surgissement de ce que l'auteur appelle, à la suite de Benjamin Harshav, une « révolution juive » dirigée contre le monde des pères ${ }^{1}$. Les Juifs contraints ou désireux de quitter l'ancien shtetl verront s'ouvrir à eux - et on tient ici la mise en perspective qui constitue l'apport majeur du livre de Slezkine - trois options. Ils pourront partir pour le Nouveau Monde, qui constitue à beaucoup d'égards une continuation de l'ancien, puisqu'il s'agit d'y exercer à une nouvelle échelle, en échappant désormais au carcan d'un régime discriminatoire, les talents du « mercurianisme traditionnel » : c'est l'option non révolutionnaire. Ils pourront choisir le sionisme et la Palestine : le projet, à l'opposé du précédent, est alors de reconfigurer un groupe mercurien à l'image des sociétés majoritaires apolliniennes, de devenir « comme les autres ». Ils pourront aussi faire un voyage, non moins long que les précédents, depuis le shtetl jusqu'aux grandes villes de la nouvelle Union soviétique, et adhérer à un modèle qui mêle traits mercuriens (l'industrialisation) et traits apolliniens (sous l'ère stalinienne, le nationalisme, l'exaltation de vertus traditionnelles).

4 L'histoire des partisans de la troisième option se découpe, comme auparavant celle des Juifs d'Europe occidentale entre les débuts de l'Émancipation et la Shoah (ou comme, pourrait-on ajouter, celle des Juifs convertis dans l'Espagne $\mathrm{du} \mathrm{xv}^{\mathrm{e}}$ siècle), en deux temps, celui de l'entrée massive dans la société soviétique et celui d'un exit symbolique puis matérialisé par l'émigration. La success story de l'intégration, dans la première période, voit les Juifs peupler l'appareil d'État et animer l'économie comme la vie culturelle (les Juifs soviétisés /russifiés ralliant les rangs de l'intelligentsia russe, puis en venant à l'incarner). Le rejet par la société majoritaire, travaillée par le nationalisme et à nouveau attachée à des valeurs "apolliniennes", est à la mesure d'une intégration ressentie comme menaçante : voilà le sens des campagnes contre les Juifs dans les dernières années du règne de Stalin - sinon contre les Juifs yiddishisants (dont la liquidation, pour Slezkine, s'inscrit en continuité avec les persécutions menées au temps de la Grande Terreur contre des groupes nationaux tenus pour potentiellement " traîtres »), du moins contre les Juifs russifiés. Le désamour ensuite des Juifs pour le régime s'expliquerait à la fois par le ressentiment face à la discrimination invisible frappant un groupe ayant particulièrement vocation à intégrer les élites soviétiques (et continuant d'ailleurs, en 
dépit de la politique de discrimination, à y être surreprésenté) et par les raisons générales qui conduisent l'intelligentsia à entrer dans la dissidence. Ce qu'il importe à Slezkine de souligner, lorsqu'il traite de la première période, c'est l'ampleur de l'adhésion au régime des Juifs menant la «révolution juive» à l'intérieur de la révolution russe. Abordant la seconde, il poursuit en priorité le parallèle avec l'histoire des Juifs (d'origine russe) aux États-Unis et celle d'Israël.

Les notations sur la vie juive américaine ne manquent pas d'intérêt. Il est éclairant, en tout cas suggestif, de qualifier l'intelligentsia new-yorkaise des années 1930 et 1940 de «fraction en exil » de l'intelligentsia juive russe. Il vaut la peine de souligner que c'est lorsque les Juifs s'américanisent pleinement qu'ils commencent à redécouvrir dans le souvenir les beautés de l'ancien shtetl, à lutter pour les Juifs d'URSS et pour Israël, bref à s'organiser en sous-groupe "national ", en conformité avec un modèle de la démocratie américaine qui invite à compenser le vide relatif d'un nationalisme purement politique par la création d'allégeances secondaires où se satisfait le besoin de participation à une histoire. En revanche, les pages consacrées à Israël ne peuvent que décevoir. Libre à l'auteur de voir dans le jeune Israël des années 1950 l'été indien du « nationalisme intégral de l'Europe de l'entre-deux-guerres», et de soutenir qu'un air de famille rapproche l'Union soviétique au tournant des années 1970, cinquante ans après la révolution d'Octobre, et l'Israël d'aujourd'hui, près de soixante ans après sa fondation (l'analogie signifiant que l'idéologie sioniste vit ses derniers jours et que l'État d'Israël est voué à se refonder en se désionisant). Mais l'inventaire des similitudes et des différences entre la culture politique du sionisme (puis d'Israël) et celle du mouvement révolutionnaire russe depuis le populisme, si nécessaire dans le cadre d'un projet qui ambitionne de confronter trois parcours historiques distincts en partant de leur surgissement au sein d'un milieu commun, est mené de façon décidément trop superficielle : mieux vaut se reporter aux pages, beaucoup plus pénétrantes, qu'Anita Shapira a consacrées au thème dans un livre rendu récemment accessible en français ${ }^{2}$.

Rien d'étonnant à ce que les Juifs aient occupé une telle place dans le jeune État soviétique, après l'élimination des anciennes élites. Ce sont les origines de la « révolution juive » qui restent largement mystérieuses : on ne les éclairera pas à coup de généralités socio-historiques sur les groupes de " nomades pourvoyeurs de services ». C'est la société juive traditionnelle au moment où elle rend l'âme qu'il eût fallu interroger.

\section{NOTES}

1. Cf. Benjamin Harshav, Language in Time of Revolution, Stanford: Stanford University Press, 1993, notamment la première partie : "The Modern Jewish Revolution : An Essay on the History of Culture and Consciousness ».

2. Anita Shapira, L'Imaginaire d'Israël. Histoire d'une culture politique, Paris: Calmann-Lévy, 2005, notamment p. 139-178: «Les dirigeants sionistes-socialistes et la révolution russe ». 